\title{
Accidentes ocupacionales en personal que labora en clínicas y consultorios de animales de compañía, Lima 2010
}

\author{
Ocupational injuries among Veterinarians in Peru \\ Jorge Breña ${ }^{1}$, Néstor Falcón ${ }^{1}$, Carla Fernández ${ }^{1}$ José Zuazo $^{1}$

\section{RESUMEN}

La práctica veterinaria es considerada como una actividad de alto riesgo y la posibilidad de sufrir daños a la salud producto de accidentes ocupacionales es latente. Objetivo: identificar los accidentes ocupacionales asociados a la actividad laboral de los profesionales que laboran en consultorios y clínicas veterinarias de animales de compañía en la ciudad de Lima durante el año 2010. Metodología: Se elaboró y aplicó una encuesta semi estructurada a Médicos Veterinarios y personal técnico que laboraban en estos lugares, para identificar datos generales de los accidentes producidos durante la actividad laboral y una percepción acerca de este problema por parte de los encuestados. Resultados y Conclusiones: Se encuestaron a 400 personas. La edad y tiempo de experiencia en la actividad fue de 29.3 (18-63) y 5.5 (1-40) años en promedio, respectivamente. El 55\% fueron varones y $45 \%$ mujeres. El $80.8 \%$ sufrieron al menos un accidente en el 2010. Los accidentes más frecuentes producidos por animales fueron mordeduras (31.1\%) y arañazos (26.6\%); y los producidos por elementos inanimados fueron pinchazos $(21.5 \%)$ y cortes $(8.7 \%)$. Los manos $(68.9 \%)$ y brazos $(20.2 \%)$ fueron las regiones anatómicas más afectadas y las cicatrices la secuela más común (49.9\%). El 44.5\% reportó problemas musculo esqueléticas en espalda, cuello y/o extremidades. El área de trabajo fue considerada cómoda para el $89 \%$ y seguras para el $79 \%$ de los trabajadores. Se hace necesario capacitar a los trabajadores de esta área sobre prácticas de seguridad y bioseguridad en el ambiente laboral.

Palabras claves: enfermedades ocupacionales, veterinarios, zoonosis.

\begin{abstract}
Veterinary practice is considered as a high-risk activity and the possibility of suffering any health injury due to occupational accidents is latent. Aim: To identify worked-related accidents in professionals who labor in animal centers and veterinary clinics of small animals in Lima city during 2010. Methods: A semi-structured survey was elaborated and applied among 400 Veterinarians and technicians who worked in these places, gathering general information of the accidents produced during work activity and the perception of this problematic among them. Results and Conclusions: The age and experience time in this type of work was 29.3 (18-63) and 5.5 (1-40) years old on average, respectively. $55 \%$ of the respondents were males and $45 \%$ females. At least $80.8 \%$ had an injury during 2010. The work-related accidents most frequently produced by animals were bites (31.1\%) and scratches (26.6\%); also lesions produced by inanimate elements were pinch (21.5\%) and cuts (8.7\%). Hands (68.9\%) and arms (20.2\%) were the anatomic regions most affected, and scars were the most commonly sequel reported (49.9\%). $44.5 \%$ of the respondents reported musculoskeletal problems on back, neck and/or extremities. The work environment was indicated as comfortable for the $89.0 \%$ of people surveyed and secure for the $79.0 \%$ of workers. There is a need to train workers in the area of environmental health and safety.
\end{abstract}

Key words: occupational diseases, veterinarians, zoonosis.

${ }^{1}$ Facultad de Medicina Veterinaria y Zootecnia - Universidad Peruana Cayetano Heredia. Lima - Perú. 


\section{INTRODUCCIÓN}

Un accidente ocupacional es aquella lesión o perturbación funcional causada en el centro laboral o durante la labor que compete a la actividad de trabajo de forma imprevista, y ocasionada por una fuerza externa que actúa sobre el profesional (Falconí, 2004). Estos inciden negativamente en el desarrollo de la actividad laboral, no estando exceptuado de ellos los profesionales del campo de la veterinaria (Álvarez et al., 2001; Reijula et al., 2003; Fritschi et al., 2006).

Casi la totalidad de profesionales veterinarios ha sufrido al menos una lesión grave durante toda su carrera o presentan lesiones crónicas relacionadas al trabajo (Fritschi et al., 2006). Las más comunes suelen ser lesiones de espalda, auto inyección accidental, mordidas, arañazos y cortes (Constable y Harrington, 1982; Hill et al., 1998; Jeyaretnam y Jones, 2000).

En profesionales que laboran con animales de compañía, las mordeduras de perro y arañazos de gato suelen se reportan como los más comunes (Jeyaretnam y Jones, 2000; Jeyaretnam et al., 2000). Las tasas más elevadas de lesiones se reportan en profesionales con 5 o menos años de experiencia o recientemente graduados (Fritschi et al., 2006; Jeyaretnam et al., 2000; Gabel y Gerberich 2002). Entre los que laboraban en zoológicos y zoocriaderos de Lima-Perú, se encontró que $60.8 \%$ había sufrido algún accidente ocupacional por instrumental o equipos, y el $85.6 \%$ sufrió ataques de animales, siendo las mordidas causadas por primates y carnívoros los más frecuentes (Lecaros et al., 2010).

En Estados Unidos se reportan lesiones en las manos como las más comunes $(52,6 \%)$, seguidas de traumas en brazos $(27,6 \%)$ y cabeza $(20,8 \%)$ : y los animales frecuentemente involucrado en fueron los vacunos $(46,5 \%)$, perros $(24,2 \%)$ y caballos $(15,2 \%)$ (Landercasper etal., 1988). En profesionales mujeres se ha reportado lesiones provocadas por agujas de jeringas con material farmacológico o biológico (vacunas, antibióticos, anestésicos y sangre de animales). La tasa de auto inoculación accidental estimada fue de 9,3 pinchazos por cada 100 personas, observándose con mayor frecuencia entre profesionales dedicados a los animales de compañía (Willkins y Bowman 1997).

Las lesiones músculo-esqueléticas se han encontrado entre 50 a $82 \%$ de Médicos Veterinarios (Fritschi et al., 2006; Cattell, 2000). Estas se encuentran relacionadas a la sujeción de los animales (Hill et al., 1998; Hafer et al., 1996), levantar a los mismos (Gabel y Gerberich 2002), cirugías prolongadas (Boyle et al., 1997), palpación rectal en ganado (Cattell, 2000; Chambers et al., 2001), movimientos repetitivos (Bernard, 1997; Frederiksson et al., 2000) stress (Devereux et al., 2004; Loomans et al., 2008; Smith et al., 2009), incomodidad o insatisfacción laboral (Smith et al., 2009; Bongers et al., 1993), tiempo y demanda del trabajo (Bernard, 1997; Bongers et al., 1993).

El personal veterinario y afines deben tener presente los riesgos de su labor y conocer en cada una de sus actividades las posibles situaciones riesgosas a las que se vería expuesto (Álvarez, 2002 ). El conocimiento de las mismas permitiría identificar las más comunes y proponer medidas de prevención para evitar o minimizar sus efectos sobre esta población. Por ello, el objetivo del estudio fue identificar y cuantificar los accidentes ocupacionales que sufrieran el personal que labora con animales de compañía en consultorios y clínicas veterinarias de Lima durante el año 2010.

\section{MATERIAL Y MÉTODOS}

Se desarrolló un estudio observacional descriptivo de corte transversal cuyo universo estaba constituido por Médicos Veterinarios y personal técnico (asistentes veterinarios, bañadores, choferes de ambulancias veterinarias) que tuvieron contacto con animales de compañía durante su actividad laboral en consultorios y clínicas veterinarias de la ciudad de Lima durante el año 2010.

El número mínimo de encuestas requeridas para el estudio fue calculado mediante la fórmula de comprobación de una proporción para poblaciones desconocidas, con un nivel de confianza del $95 \%$, un error máximo admisible del $5 \%$ y una proporción de profesionales afectados por accidentes del $50 \%$. El tamaño de muestra fue de 385 personas a las que encuestarían entre Médicos Veterinarios y personal técnico. Los mismos fueron seleccionados mediante un muestreo por conveniencia y por agotamiento. El trabajo de campo se realizó en el periodo comprendido entre Junio y diciembre de 2010.

La participación en el estudio fue estrictamente voluntaria, por lo que los participantes confirmaron su participación con la firma de un consentimiento informado. El estudio contó con el permiso del Comité Institucional de Ética de la UPCH. La información proporcionada por los participantes fue manejada de forma absolutamente confidencial y anónima. 
Para la recolección de la información se elaboró una encuesta semiestructurada (preguntas abiertas y cerradas), la que se encontraba dividida en dos partes. La primera correspondió a datos generales del participante (lugar donde labora, labor que realiza, condición laboral, edad, sexo, años de experiencia) y la segunda, información de accidentes y riesgos ocupacionales (accidentes ocupacionales, exposición a químicos, lesiones musculares y óseas o articulares). La validación del instrumento se realizó en la Clínica Veterinaria de la Facultad de Medicina Veterinaria y Zootecnia de la Universidad Peruana Cayetano Heredia.

La recolección de datos se realizó a través de visitas a consultorios y clínicas veterinarias de la ciudad de Lima. Las encuestas fueron leídas y llenadas por los encuestados; el investigador estuvo presente con el fin de absolver cualquier duda y evitar errores de interpretación y llenado de las encuestas.

La información obtenida a partir de las encuestas fue transferida a una base de datos en el programa Microsoft Excel y tras la revisión completa de la misma se obtuvo la base de datos definitiva para realizar el análisis estadístico utilizando el programa SPSS 17.0. Los datos fueron resumidos mediante tabla de frecuencias. Las variables cuantitativas se resumieron mediante medida de tendencia central (media) y medidas de dispersión (la desviación estándar y rango).

\section{RESULTADOS}

Se realizaron 400 encuestas entre Médicos Veterinarios (MV) y personal técnico (PT) que laboraban en los diferentes consultorios y clínicas veterinarias de la ciudad de Lima-Perú. Del total de encuestados el $39.5 \%$ fueron MV (158) y el $60.5 \%$ PT (242). Los encuestados tuvieron un promedio de edad de 29.3 años (desvió estándar $=7,45$ y rangos de 18,0 a 63,0 años). El tiempo de experiencia promedio en la actividad fue de 5.5 años (desvió estándar = 5.47 años y rangos de 1.0 a 40.0 años). El 55\% (220) fueron de sexo masculino (92 MV y $128 \mathrm{PT}$ ) y el $45 \%$ (180) de sexo femenino (66 MV y 114 PT). Del PT, el $32.3 \%$ (129) eran asistentes veterinarios, $17.3 \%$ (69) bañadores y $11.0 \%$ (44) realizaban otras labores (choferes, recepción de animales, entre otros).

El 80.8\% (323) del personal que laboran en clínica de animales de compañía sufrieron al menos un accidente en el último año. En varios casos, el personal laboral había sufrido más de un accidente haciendo un total de 702 accidentes de los cuales el 46.4\% (326) fueron reportados entre MV y 53.6\% (376) en PT.

Los accidentes que se reportaron con mayor frecuencia fueron mordeduras, arañazos y pinchazo o inyección sin fluido. Este último fue lo más común entre los MV y las mordeduras entre el PT. La distribución de los accidentes se presenta en el cuadro 1. En el cuadro 2 se muestra la distribución de las lesiones.

De todos los episodios reportados solo 28 (4.0\%) fueron calificados como graves (16 en MV y 12 en PT), los 674 (96.0 \%) restantes fueron considerados por los encuestados como accidentes leves (310 en MV y 364 en PT). Además, 515 (73.4\%) eventos no requirió de ningún tipo de atención médica (220 en MV y 295 en PT), 137 (19.5\%) se auto medicaron (93 MV y 44 PT) y tan solo en 50 casos (7.1\%) requirió de asistencia médica (13 MV y $37 \mathrm{PT}$ ).

En relación a los días que se vieron imposibilitados de realizar sus labores, en 658 accidentes (93.7\%) no se afectó la rutina de trabajo (322 en MV y 336 en PT), mientras que en $24(3.4 \%)$ accidentes no se trabajó un día (1 MV y 23 PT) y 11 (1.6\%) por dos días (1 MV y $10 \mathrm{PT}$ ). Se observa que las regiones anatómicas más afectadas fueron las manos y brazos tanto en MV como en PT. El cuadro 3 muestra las secuelas de estos accidentes, donde las cicatrices son las más frecuentes en ambos grupos laborales, y el cuadro 4 muestra la distribución de los implementos de protección personal utilizados durante las actividades de trabajo por personal que labora en clínicas y consultorios de animales de compañía.

En cuanto a problemas musculo esqueléticas (de espalda, cuello y extremidades), estas fueron reportados por el 44.5\% (178) de los encuestados (76 MV y 102 PT). Del total, 74,2\% (132) reportaron haber sufrido problemas de espalda (60 MV y $72 \mathrm{PT})$, $35.4 \%$ (63) en cuello (30 MV y $33 \mathrm{PT}$ ) y $54.5 \%$ (72) en extremidades (24 MV y $48 \mathrm{PT}$ ). De estos, $17.4 \%$ (23), 35.4\% (63) y $2.8 \%$ (Álvarez et al., 2001) de los problemas musculo esqueléticos en espalda, cuello y extremidades, lo consideraron como grave.

Del total de encuestados que mencionaron haber sufrido lesiones musculo esqueléticas 26.4\% (47) recibieron atención médica (18 MV y $29 \mathrm{PT}$ ); 50.6\% (90) se auto medicaron (31 MV y 59 PT) y el restante $23.0 \%$ (41) no recibieron atención médica ni se auto medicaron (27 MV y 24 PT). Las lesiones musculo 
Cuadro 1. Distribución de los tipos de accidentes según personal que labora en clínicas y consultorios de animales de compañía. Lima - Perú, 2010.

\begin{tabular}{lllllll}
\hline \multirow{2}{*}{\multicolumn{1}{c}{ Tipo de Accidente }} & \multicolumn{2}{l}{ Médicos } & \multicolumn{2}{l}{ Personal } & \multicolumn{2}{l}{ Técnico } \\
& \multicolumn{2}{l}{ Veterinarios } & \multicolumn{2}{l}{$\begin{array}{l}\text { Total de } \\
\text { encuestados }\end{array}$} \\
& Nro. & $\%$ & Nro. & $\%$ & Nro. & $\%$ \\
\hline Mordeduras & 83 & 25.5 & 135 & 35.9 & 218 & 31.1 \\
Arañazos & 80 & 24.5 & 107 & 28.4 & 187 & 26.6 \\
Pinchazo o inyección sin fluido & 100 & 30.7 & 51 & 13.6 & 151 & 21.5 \\
Cortes & 30 & 9.2 & 31 & 8.2 & 61 & 8.7 \\
Pinchazo o inyección con fluido & 20 & 6.1 & 15 & 4.0 & 35 & 5.0 \\
Lesiones con caniles & 9 & 2.8 & 12 & 3.2 & 21 & 3.0 \\
Otros & 4 & 1.2 & 15 & 4.0 & 19 & 2.7 \\
Quemaduras & 0 & 0.0 & 10 & 2.7 & 10 & 1.4 \\
TOTAL & 326 & 100.0 & 376 & 100.0 & 702 & 100.0 \\
\hline
\end{tabular}

Cuadro 2. Ubicación anatómica de las lesiones causadas por los accidentes en personal que labora en clínicas y consultorios de animales de compañía. Lima - Perú, 2010.

\begin{tabular}{|c|c|c|c|c|c|c|}
\hline \multirow{2}{*}{ Ubicación Anatómica } & \multicolumn{2}{|c|}{$\begin{array}{l}\text { Médicos } \\
\text { Veterinarios }\end{array}$} & \multirow{2}{*}{$\begin{array}{l}\text { Personal } \\
\text { Nro. }\end{array}$} & \multirow{2}{*}{$\begin{array}{l}\text { Técnico } \\
\%\end{array}$} & \multicolumn{2}{|c|}{$\begin{array}{l}\text { Total de } \\
\text { encuestados }\end{array}$} \\
\hline & Nro. & $\%$ & & & Nro. & $\%$ \\
\hline Manos & 258 & 79.2 & 225 & 59.7 & 483 & 68.9 \\
\hline Brazos & 35 & 10.7 & 107 & 28.5 & 142 & 20.2 \\
\hline Manos y brazos & 6 & 1.8 & 16 & 4.3 & 22 & 3.1 \\
\hline Cuello & 10 & 3.1 & 1 & 0.3 & 11 & 1.6 \\
\hline Cabeza & 8 & 2.5 & 2 & 0.5 & 10 & 1.4 \\
\hline Espalda & 2 & 0.6 & 8 & 2.1 & 10 & 1.4 \\
\hline Tórax & 1 & 0.3 & 6 & 1.6 & 7 & 1.0 \\
\hline Piernas & 4 & 1.2 & 3 & 0.8 & 7 & 1.0 \\
\hline Pies & 0 & 0.0 & 7 & 1.9 & 7 & 1.0 \\
\hline Otros & 2 & 0.6 & 1 & 0.3 & 3 & 0.4 \\
\hline TOTAL & 326 & 100.0 & 376 & 100.0 & 702 & 100.0 \\
\hline
\end{tabular}

Cuadro 3. Distribución de las secuelas de accidentes en personal que laboran en clínicas y consultorios de animales de compañía. Lima - Perú, 2010.

\begin{tabular}{|c|c|c|c|c|c|c|}
\hline \multirow{2}{*}{ Secuelas del Accidente } & \multicolumn{2}{|c|}{$\begin{array}{l}\text { Médicos } \\
\text { Veterinarios }\end{array}$} & \multirow{2}{*}{$\begin{array}{l}\text { Personal } \\
\text { Nro. }\end{array}$} & \multirow{2}{*}{$\begin{array}{l}\text { Técnico } \\
\%\end{array}$} & \multicolumn{2}{|c|}{$\begin{array}{l}\text { Total de } \\
\text { encuestados }\end{array}$} \\
\hline & Nro. & $\%$ & & & Nro. & $\%$ \\
\hline Cicatrices & 136 & 41.7 & 214 & 56.9 & 350 & 49.9 \\
\hline Dolor crónico leve & 40 & 12.3 & 41 & 10.9 & 81 & 11.5 \\
\hline Otros & 7 & 2.2 & 27 & 7.2 & 34 & 4.8 \\
\hline Infección & 4 & 1.2 & 5 & 1.3 & 9 & 1.3 \\
\hline Dificultad de movimiento & 3 & 0.9 & 4 & 1.1 & 7 & 1.0 \\
\hline Dolor crónico moderado & 3 & 0.9 & 1 & 0.3 & 4 & 0.6 \\
\hline TOTAL & 326 & 100.0 & 376 & 100.0 & 702 & 100.0 \\
\hline
\end{tabular}


Cuadro 4. Distribución de los Implementos de protección personal utilizados durante las actividades de trabajo por personal que labora en clínicas y consultorios de animales de compañía. Lima - Perú, 2010.

\begin{tabular}{lllllll}
\hline $\begin{array}{l}\text { Implementos de protección personal } \\
\text { utilizados durante las labores }\end{array}$ & \multicolumn{2}{l}{$\begin{array}{l}\text { Médicos Veterinarios } \\
(\mathrm{n}=158)\end{array}$} & $\begin{array}{l}\text { Personal } \\
(\mathrm{n}=242)\end{array}$ & Técnico & \multicolumn{2}{l}{$\begin{array}{l}\text { Total de } \\
\text { encuestados }(\mathrm{n}=400)\end{array}$} \\
\cline { 2 - 7 } Mandil & Nro. & $\%$ & Nro. & $\%$ & Nro. & $\%$ \\
Guantes & 143 & 90.5 & 156 & 64.5 & 299 & 74.8 \\
Mascarilla & 153 & 96.8 & 145 & 59.9 & 298 & 74.5 \\
Lentes & 131 & 82.9 & 132 & 54.6 & 263 & 65.8 \\
Ninguno & 29 & 18.4 & 32 & 13.2 & 61 & 15.3 \\
Otro & 1 & 0.6 & 50 & 20.7 & 51 & 12.8 \\
\hline
\end{tabular}

esqueléticas imposibilitaron desarrollar normalmente sus actividades a $22.5 \%$ (40) de los afectados (20 MV y $20 \mathrm{PT}$ ) entre 1 y 5 días. Solo el $0.56 \%$ (1PT) se vio imposibilitado de realizar sus labores por un periodo de entre 5 y 10 días. Los demás no dejaron de laborar por esta causa.

El área de trabajo fue considerada cómoda para el $89.0 \%$ (356) de los encuestados (141 MV y 215 PT) y seguras para el $79.0 \%$ (316) de los trabajadores (116 MV y 200 PT). El 100\% de MV consideró importante una mayor capacitación para el personal que labora en clínicas y consultorios veterinarios en contraste con el PT quienes solamente el 2.8\% consideró necesario una mayor capacitación.

\section{DISCUSIÓN}

Las personas que laboran en cualquier actividad se encuentran expuestas a riesgos laborales capaces de ocasionar daño o afectar su salud. La escasa información que existe respecto a los accidentes ocupacionales origina que los trabajadores no estén conscientes de los riesgos a los que están potencialmente expuestos. En este contexto, las labores dentro de la clínica de animales de compañía son particularmente riesgosas debido a que el personal se encuentra en constante contacto con animales. El estudio incluye accidentes producidos solamente en el ambiente laboral, es decir en el desarrollo de una actividad dentro del horario normal de dicha actividad (Funes, 1979 ).

El estudio encuentra que cuatro de cada cinco personas que laboraban en clínicas o consultorios veterinarios habrían sufrido por lo menos un accidente durante su actividad laboral, en el año 2010. El periodo de estudio estuvo limitado a este tiempo con la finalidad de evitar el sesgo de memoria que podría llevar a subestimar algunos resultados debido a que los encuestados ya no recordaban de algunos eventos producidos en periodos alejados a la actualidad.

Las lesiones más frecuentes producidas por animales fueron mordidas y arañazos. Estos suelen suceder generalmente por descuidos en la sujeción de los mismos. Estos accidentes se presentaron con mayor frecuencia en el personal técnico debido a que ellos están en mayor contacto con el animal, porque suelen ser los encargados de la sujeción durante la atención veterinaria y otras actividades (baño, transporte, contención, etc.). Estas reacciones se producen porque el consultorio o clínica veterinaria es un ambiente extraño para los animales, pudiéndose sentir amenazados con lo que las muestras de agresividad representan un mecanismo de defensa. Estudios similares realizados en Chile (Cam, 2005; Deck, 2003) encuentran también que mordeduras y arañazos son los accidentes más frecuentes en este tipo de práctica veterinaria.

Entre los accidentes producidos por objetos inanimados, la auto inyección y cortes fueron los accidentes más comunes. En ambos casos, estos se producían durante el tratamiento de pacientes que se movieron súbitamente. Las auto inyecciones también se produjeron durante la manipulación de agujas y jeringas durante la preparación de los inyectables que se administrarían a los pacientes, por ello se observan con alta frecuencia las auto-inyecciones sin fluidos. Este tipo de accidentes se presentó con mayor frecuencia entre Médicos Veterinarios debido a que son ellos los encargados de preparar y administrar las inyecciones. Estudios realizados en diferentes países (Constable y Harrington, 1982; Hill et al., 1998; Jeyaretnam y Jones, 2000; Jeyaretnam et al., 2000; Landercasper et al., 1988), así como un estudio realizado en el Perú con profesionales que trabajaban 
con animales silvestres (Lecaros et al., 2010), reportan resultados similares.

La importancia de estos tipos de accidentes se refleja en el trauma sufrido y por la secuela que se pudiera producir (infecciones, transmisión de enfermedades, inhabilitación temporal del miembro afectado, cicatrices, etc.). Se menciona que este tipo de accidentes son comunes en veterinarios debido a que tratan pacientes estresados y de comportamiento impredecible (Willkins y Bowman 1997).

Las zonas anatómicas generalmente afectados fueron las manos y brazos. Ello se explicaría por el hecho de que estas regiones anatómicas se encuentran más cerca del hocico y garra de los animales durante la manipulación de los mismos. Además, estos miembros suelen ser la primera barrera de defensa de las persona cuando los animales se encuentran en libertad para atacar. La evaluación entre Médicos Veterinarios y cuidadores de zoológicos y zoocriaderos, encontró resultados y sustentaciones similares a la presencia de estas regiones anatómicas afectadas (Lecaros et al., 2010).

En la mayoría de accidentes, por causa animales o instrumental, los afectados consideraron que los que fueron de carácter leve por lo que no requirieron visitar al Médico, y en muchos de los casos se auto medicaron. Ahorrar tiempo o dinero, además de evitar la pérdida del día de trabajo, justifican en muchas ocasiones esta práctica.

Los elementos de protección utilizados con mayor frecuencia en la práctica laboral entre los trabajadores de los consultorios y clínicas veterinarias fueron mandil, guantes y mascarilla. Sin embargo se debe de considerar que estos implementos son los básicos y mínimos para la atención y debería de ser utilizado por el $100 \%$ de los profesionales de esta área. Estos implementos de protección van a evitar en cierto nivel la exposición, especialmente a microorganismos potencialmente patógenos y sustancias irritantes, pero no evitaran lesiones en casos de accidentes con objetos punzo cortantes o ataques de animales.

La presencia de problemas musculo esqueléticas son elevadas en el estudio (más del 40.0\%). Se menciona que estos problemas son la mayor causa de dolor y lesiones, enfermedades, reducción de la productividad y ausentismo en el trabajo y que incrementan los costos de personal (Buckle, 2005; MacDonald, 2004; Kourinka, 1995 ). Los problemas musculo esqueléticos no son un fenómeno nuevo, ya que estos han sido reconocidos desde tiempos antiguos por Ramazzini, considerado el padre de la medicina ocupacional (Franco, 2004 ).

A las lesiones musculo esqueléticas se les da poca importancia entre los Médicos Veterinarios, aunque estas se encuentran una alta prevalencia en este grupo profesional (Cam, 2005). La sujeción, el levantar los animales, posturas estáticas prolongadas, movimientos repetitivos, tiempo y demanda del trabajo (cirugías prolongadas), mobiliario inadecuado, entre otros, se reconoce como factores que favorecen la presentación de estos problemas. Durante las cirugías, el Médico Veterinario debe permanecer largas horas de pie, con la presión de mantener el control sobre la situación, por lo que es indispensable, para evitar problemas de dolor de cuello, espalda y de extremidades, tener una mesa quirúrgica regulable en altura.

La percepción mayoritaria de los trabajadores es que su área de trabajo es cómoda y segura. Sin embargo en muchas ocasiones el trabajador se acondiciona al área de trabajo, acostumbrándose al mismo. A fin de que se mejoren las condiciones de trabajo, el mobiliario, como mesa de atención, de cirugía, de peluquería, entre otros, debería ser regulable a fin de quien las use la adecue a su estatura.

La capacitación del personal que labora en clínicas y consultorios veterinarios es un aspecto que todos los Médicos Veterinarios consideran como muy importante e imprescindible a fin de disminuir los riesgos a los que están expuestos los trabajadores de clínicas y consultorios veterinarios. Sin embargo, un pequeño grupo del personal técnico considera que no es necesaria la capacitación y que todo lo que se requiere se aprende con la práctica, con lo que los accidentes son considerados parte del aprendizaje en el trabajo.

A la luz de los resultados se puede concluir que la salud ocupacional es un tema de importancia dentro del ámbito veterinario, no solo desde el punto de vista médico sino también por el confort que debe tener cualquier trabajador para desarrollar su actividad. Se considera que un ambiente de trabajo saludable permitirá un mejor desempeño de las capacidades de los trabajadores y un aumento del beneficio económico, reduciendo días de trabajo perdidos o disminuyendo costos de atención médica. Así mismo, el ambiente de trabajo mismo y la organización de este, además de las condiciones y técnicas laborales, repercuten 
en el desarrollo del trabajo y afectan la salud física y mental de los trabajadores. Por ello, también es responsabilidad de cada trabajador, velar por que en su entorno laboral se implementen las mejores condiciones posibles que minimicen los accidentes ocupacionales.

\section{CONCLUSIONES}

- La mayoría de Médicos Veterinarios y personal técnico que laboran en Clínicas y Consultorios veterinarios de Lima, han sufrido por lo menos un accidente ocupacional durante sus labores el año 2010, siendo los más frecuentes las mordidas y los arañazos y los pinchazos por inyección sin fluido.

- El los Médicos Veterinarios los accidentes por pinchazos y por inyección sin fluido fueron los más frecuentes, mientras que en el personal técnico los accidentes por mordidas, se reportan en mayor frecuencia. En ambos casos las zonas del cuerpo más afectadas son las manos y los brazos, y las secuelas más comunes fueron las cicatrices.

- Los problemas musculoesqueléticas en espalda, cuello o extremidades, fueron reportados con mayor frecuencia por los Médicos Veterinarios, siendo los problemas de cuello los más comunes.

\section{Correspondencia:}

Néstor Falcón

nestor.falcon@upch.pe

\section{REFERENCIAS BIBLIOGRÁFICAS}

1. Álvarez R. (2002). Salud pública y medicina preventiva. México: El Manual Moderno.

2. Álvarez E, Larrieu L, Cavagión L, García M. (2001). Riesgos Ocupacionales de los profesionales veterinarios y trabajadores rurales con animales. Aplicación y enseñanza. Facultad de Ciencias Veterinarias. U.N.L. p. 52-62.

3. Bernard B. (1997). Musculoskeletal disorders and workplace factors. Cincinnati, USA, National Institute of occupational safety and health (NIOSH). Disponible en: http://www.cdc.gov/niosh/docs/97141/pdfs/97-141.pdf

4. Bongers PM, De Winter C, Kompier M, Hildebrndt V. (1993). Psychosocial factors at work and musculoskeletal disease. Scandinavian journal of work, environment \& health 19: 297-312.

5. Boyle D, Gerberich SG, Gibson RW, Maldonado G, Robinson AR, Martin F, Renier C. Amadeus H. (1997). Injury from dairy cattle activities. Epidemiology 8:37-41.

6. Buckle P. (2005). Ergonomics and musculoskeletal disorders: overview. Occupational medicine 55: 164167.
7. Cam Z. (2005). Estudio de los riesgos laborales asociados al ejercicio de la profesión de médico veterinario en clínicas veterinarias de Pequeños animales de las ciudades de la serena y coquimbo, IV Región Chile. Tesis licenciado en Ciencias Veterinarias. Universidad Católica de Temuco, Chile.

8. Cattell, M. B. (2000). Rectal palpation associated cumulative trauma disorders and acute traumatic injury affecting bovine practitioners. Bovine practitioner 34:1-5.

9. Chambers M, Cover A, Duczmal T, Mc Grath K. (2001). Healthy, wealthy and wise-AACV Healthy and lifestyle survey 2001. In Australian association of cattle veterinarians Dubbo, Australian association of cattle veterinarians p. 42-51.

10. Constable P, Harrington J. (1982). Risks of zoonoses in a veterinary service. Br Med J 284: 246-248.

11. Deck E. (2003). Estudio de Riesgos Laborales en Médicos Veterinarios del Área de Clínica de Pequeños Animales en las Ciudades de Concepción y Talcahuano y comunas de San Pedro de la Paz y Chiguayante. Tesis de Médico Veterinario. Universidad Católica de Temuco, Chile.

12. Devereux J, Rydstedt L, Kelly V, Weston P, Buckle P. (2004). The role of work stress and psychological factors in development of musculoskeletal disorders: The stress and MSD study. Guilford, Health and safety executive. Reserch report 273. 153p.

13. Falconí R. (2004). Enfermedades Profesionales, Accidentes de trabajo y seguro complementario de trabajo de riesgo. Revista de la Academia Peruana de Salud: Disponible en: http://sisbib.unmsm.edu.pe/ bvrevistas/rev_academia/2004_n1/pdf/a05.pdf

14. Franco G, Fusetti L. (2004). Bernardino Ramazzini's early observations of the link between musculoskeletal disorders and ergonomic factors. Applied Ergonomics 35:67-70.

15. Frederiksson K, Alfredsson L, Thorbjornsson CB, Punnet L, Toomingas A, Torgen M, Kilbom A. (2000). Risk factors for neck and shoulder disorders: a nested case-control study covering a 24 year period. American journal of industrial medicine 38: 516-528.

16. Fritschi L, Day L, Shirangi A, Robertson I, Lucas M, Vizard A. (2006). Injury in Australian veterinarians. Occup Med 56: 199-203

17. Funes G. (1979). Curso de Seguridad Básica, obreros Mina. Codelco, Chile, División El Teniente; Dpto. Prevención de Riesgos.

18. Gabel C, Gerberich S. (2002). Risk Factors for Injury among Veterinarians. Epidemiology 13: 80-86.

19. Hafer A, Langley RL, Morrow WE, Tulis JJ. (1996). Occupational hazards reported by swine veterinarians in the United States. Swine helth production 4:128141.

20. Hill D, Langley R, Morgan W. 1998. Occupational Injuries and Illnesses Reported by Zoo Veterinarians in the United States. J Zoo Wildl Med 29: 371-385.

21. Jeyaretnam J, Jones H, Phillips M. (2000). Disease 
and injury among veterinarians. Aust. Vet. J. 78(9):625-9.

22. Jeyaretnam J, Jones H. (2000). Physical, chemical and biological hazards in veterinary practice. School of Public Health. Aust Vet J 78: 751-758.

23. Kourinka I, Forcier L, Hagberg M. (1995). Work related Musculoskeletal disorders (WMSDs): A reference book for prevention, Taylor \& Francis limited, London.

24. Landercasper J, Cogbill T, Strutt P, Landercasper B. (1988). Trauma and the Veterinarian. The J Trauma 28: $1255-1259$.

25. Lecaros A, Falcón N, Elías R. (2010). Accidentes ocupacionales y zoonosis en médicos veterinarios $\mathrm{y}$ cuidadores de zoológicos y zoocriaderos de la ciudad de Lima - Perú. Revista Sapuvet de Salud Pública 2: 27-42.

26. Loomans JBA, Van Weeren-Bitterling MS, Van Weeren PR, Barneveld A. (2008). Occupational disability and job satisfaction in the equine veterinary profession: How sustainable is this "Tough job" in a changing world? Equine veterinary Education 2008; 20: 597-607.
27. Mac Donald W. (2004). Workload, stress and psychosocial factors as hazards for musculoskeletal disorders. Journal of occupational health and safety, Australian and New Zeland 20: 37-47.

28. Reijula K, Rasanen K, Hamalainen M, Juntunen K, Lindbohm ML, Taskinen H, et al. (2003). Work environment and occupational health of Finnish Veterinarians. Am J Ind Med 44: 46-57.

29. Smith DR, Leggat PA, Speare R. (2009). Musculoskeletal disorders and psychosocial risk factors amoung veterinarians in Queensland, Australia. Australian veterinary journal 87(7): 260265.

30. Willkins J, Bowman M. (1997). Needlestick injuries among female veterinarians: frequency, syringe contents and side-effects. Occup Med 47: 8,451-457.

Recibido: 22/05/2014

Aceptado:17/06/2014 